Check for updates

Cite this: RSC Adv., 2017, 7, 48308

Received 12th July 2017

Accepted 9th October 2017

DOI: $10.1039 / \mathrm{c} 7 \mathrm{ra0} 07665 \mathrm{~g}$

rsc.li/rsc-advances

\section{Optically observed multiple inter-chain interactions in polyblend semiconducting polymer nanoparticles}

\begin{abstract}
Z. Hashim, (D) *a S. Alomari, ${ }^{a}$ W. Alghamdi, ${ }^{a}$ R. Altuwirqi ${ }^{a}$ and M. Green (D) ${ }^{\text {b }}$
F8BT:MEH-PPV:CN-PPV semiconducting polymer blend nanoparticles (SPNs) were synthesized with different ratios by a miniemulsion route from three different conjugated polymers; two red/orange emitting polymers; poly[2-methoxy-5-(2-ethylhexyloxy)-1,4-phenylenevinylene] (MEH-PPV) and poly(2,5-di(hexyloxy)cyanoterephthalylidene) (CN-PPV), and one green emitting polymer poly(9,9dioctylfluorene-alt-benzothiadiazole) (F8BT). The resulting SPNs were spherical in shape as determined by transmission electron microscopy, and exhibited tuneable photoluminescence. These optical properties were attributed to the inter-chain interactions between F8BT and the MEH-PPV, and between MEH-PPV and CN-PPV within the same nanoparticle, with no direct interactions happening between the chains of F8BT and the chains of CN-PPV. Therefore, the presence of MEH-PPV within the polyblend SPNs facilitated an indirect F8BT:CN-PPV interaction that was not possible before.
\end{abstract}

\section{Introduction}

Semiconducting polymer nanoparticles (SPNs) are fluorescent materials which are proposed as alternatives to inorganic quantum dots. ${ }^{1}$ SPNs have better fluorescence stability with longer shelf-lives, are composed of benign or less-toxic materials, and can be synthesized by an environmentally friendly and relatively easy method (miniemulsion) that does not require any heating, and can be performed in ambient conditions. Many applications require fluorescence within a specific range of wavelengths, selected for the relevant end-use. ${ }^{2}$ Nanoparticles engineered for these applications must, therefore, have stable emission that does not change or quench in their intended environment over the period of storage and use. Unlike quantum dots, ${ }^{3,4}$ SPNs have emission colors that do not shift with the change in their size..$^{5-7}$ However, if a different emission color is required, the SPNs' parent polymer must be changed or a dopant molecule ${ }^{8}$ or further polymer ${ }^{9}$ must be introduced within the same nanoparticle during the synthesis process to facilitate inter-chain interactions that result in the required fluorescence change. A key method for tuning the optical properties of SPNs is by preparing polymer blends prior to nanoparticle formation, resulting in what can be referred to as polyblend SPNs. ${ }^{10}$

Several studies reported the synthesis of polyblend SPNs from a blend of two conjugated polymers; ${ }^{11,12}$ Huebner et al. ${ }^{12}$

${ }^{a}$ Department of Physics, Faculty of Sciences, King Abdulaziz University, Jeddah, Saudi Arabia. E-mail: zmhashim@kau.edu.sa

${ }^{b}$ Department of Physics, King's College London, Strand, London, WC2R 2LS, UK. E-mail:mark.a.green@kcl.ac.uk reported the synthesis of PFO:POPPV SPNs from a blend of a blue emitting (PFO) and a green emitting (POPPV) polymer. The blending resulted in a material with controllable photoemission caused by the tunable energy transfer within the nanoparticles. This energy transfer was also reported by McNeill et al. ${ }^{11}$ when blending F8BT with MEH-PPV. The blend caused the total quenching of F8BT emission and an increase in the optical quantum yield of the new MEH-PPV-emitting polyblend SPNs. Color control of polyblend SPNs could pave the way towards the production of white emitting nanoparticles from a blend of polymers with different emission colors, ${ }^{13}$ while fluorescence emission enhancement of pre-existing polymer nanoparticles by doping them with trace amounts of other polymers could increase their emission efficiency. ${ }^{14}$ Some polyblend SPNs were also found to have multiple electron densities within one nanoparticle. This phase separation phenomena can be utilized to form core/shell structures similar to those of QDs. ${ }^{13}$

In this study, we report the synthesis of new polyblend SPNs synthesized from the blend of three conjugated polymers; F8BT, MEH-PPV, and CN-PPV (polymer structures shown in Fig. 1). The new F8BT:MEH-PPV:CN-PPV SPNs have controllable optical absorption and tunable emission which can be altered by the modification of the polymers' ratios in the SPNs.

\section{Results and discussion}

Fig. 2 shows TEM images of the prepared F8BT:MEH-PPV:CNPPV SPNs. The synthesized nanoparticles were mostly spherical and their sizes ranged between tens of nanometers and a few hundred nanometers in diameter (the diameter size 


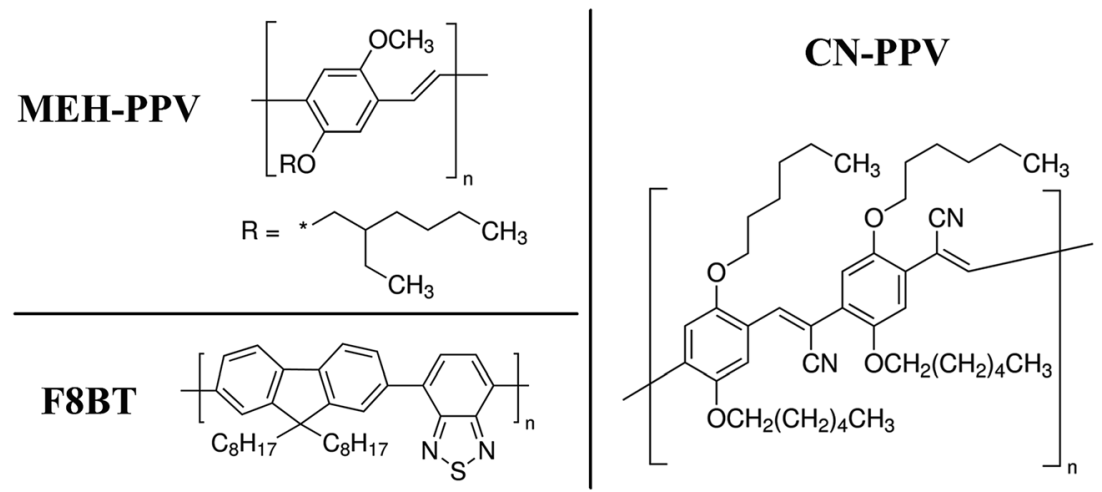

Fig. 1 The chemical structures of MEH-PPV, F8BT, and CN-PPV.

distribution of a typical sample is shown in Fig. 3, mean diameter $=90.6 \mathrm{~nm}$ ). This broad particle range is expected from a miniemulsion synthesis process, and can be minimized with further emulsion control. ${ }^{5}$ The SDS coating was also visible especially around the larger particles (Fig. 2C, E, and F). Fig. 2C and $\mathrm{D}$ also show uneven electron density within the same particle, previously assigned as phase separation in blended polymer nanoparticles. ${ }^{15}$

The absorption spectra of F8BT:MEH-PPV:CN-PPV SPNs and of SPNs prepared from the individual polymers are shown in Fig. 4A. The peaks of the absorption spectra of F8BT:MEHPPV:CN-PPV SPNs in the visible region, i.e. between 390$700 \mathrm{~nm}$, overlaid those of both CN-PPV SPNs and F8BT SPNs, but their widths varied between both nanoparticles. The second absorption peaks of F8BT:MEH-PPV:CN-PPV SPNs, which appeared in the ultraviolet region, i.e. between $375-390 \mathrm{~nm}$, followed the shape of F8BT SPNs but had lower, variable, intensities.
The emission spectra of F8BT:MEH-PPV:CN-PPV SPNs and of SPNs prepared from the individual polymers are shown in Fig. 4B. The nanoparticles prepared from the blend of the three polymers had only one emission peak between 500-750 nm which was similar to that of CN-PPV SPNs and MEH-PPV SPNs, with its peak value shifting between the peak values of the two red emitting polymer SPNs, and its emission width widening to cover both spectra. Moreover, although the presence of F8BT was suggested by the absorption spectra, there was no sign of the existence of the fluorescence of F8BT in the nanoparticles prepared with low ratios of F8BT (which should appear as a second peak or a shoulder around $\lambda_{\mathrm{em}}=536 \mathrm{~nm}$ ), and there was only a small shoulder at that wavelength in the SPNs prepared with the highest $\mathrm{F} 8 \mathrm{BT}$ ratio (i.e. $60: 20: 20 \mathrm{SPNs}$ ). F8BT is known to have a substantially higher fluorescence quantum yield than MEH-PPV, ${ }^{16}$ and the existence of only a small shoulder around its emission peak of $60: 20: 20$ SPNs and its total disappearance, in the samples with lower F8BT
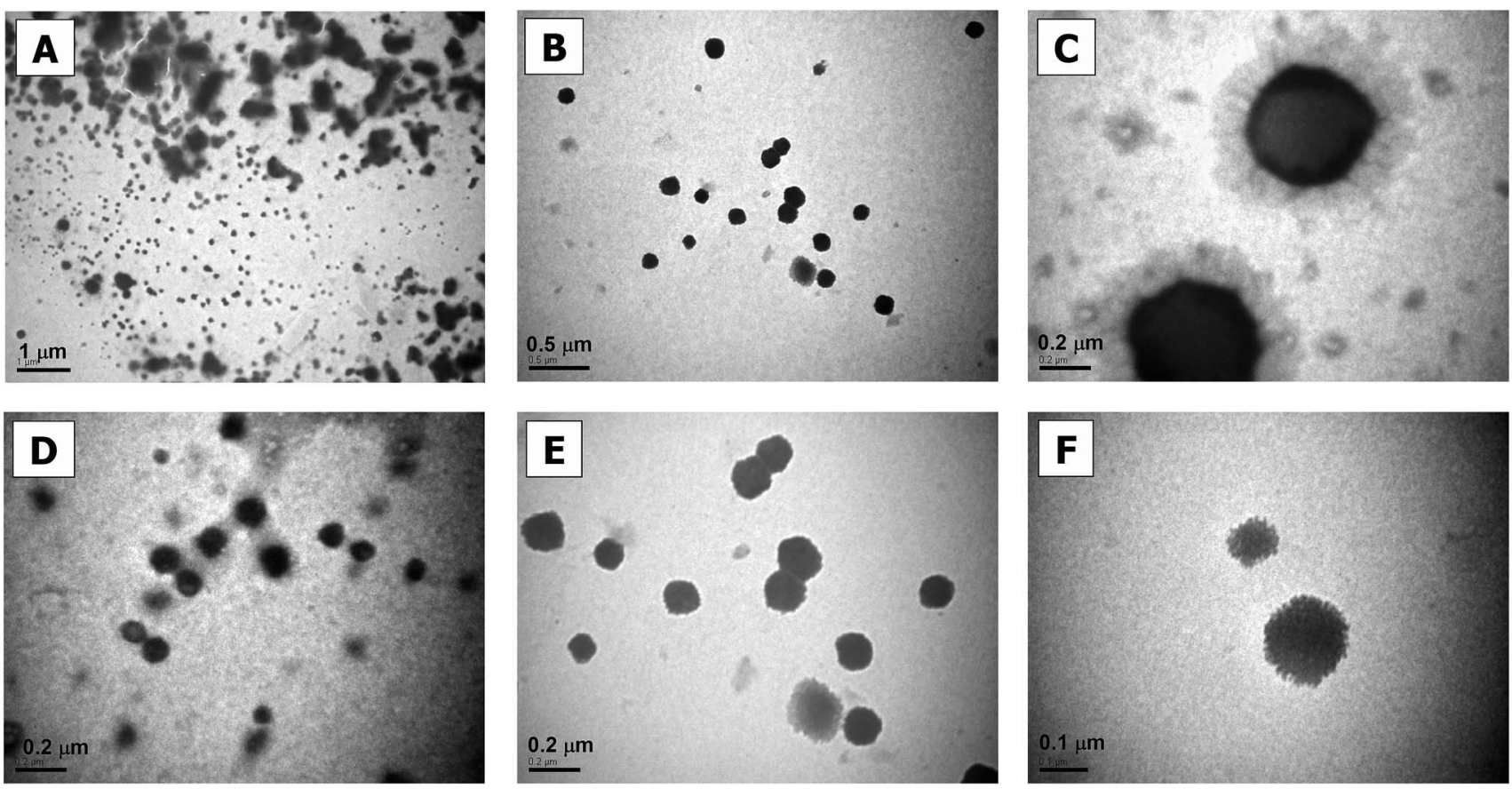

Fig. 2 TEM images of F8BT:MEH-PPV:CN-PPV SPNs. Bar-scales are $1 \mu \mathrm{m}$ in (A), $0.5 \mu \mathrm{m}$ in (B), $0.2 \mu \mathrm{m}$ in (C-E), and $100 \mathrm{~nm}$ in (F). 


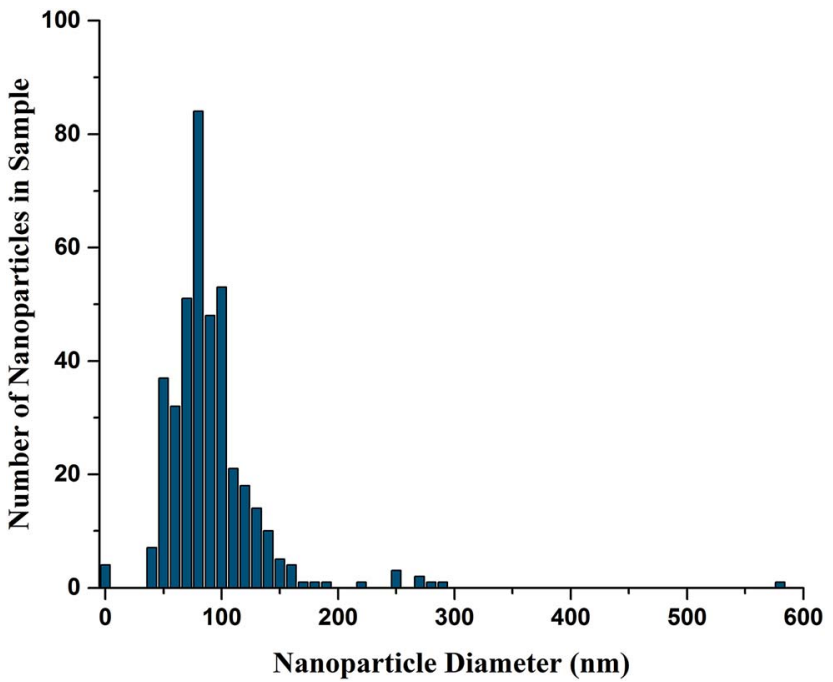

Fig. 3 F8BT:MEH-PPV:CN-PPV SPNs diameter size distribution, measured from TEM images, 400 counts, using ImageJ.

ratios, suggest that there was an interaction between F8BT and at least one of the other polymers.

To investigate if this behavior was due to the compact configuration of the three polymers in one nanoparticle or merely due to the existence of the polymers in separate nanoparticles in the same solution, mixtures of pre-synthesised MEH-PPV SPNs, F8BT SPNs, and CN-PPV SPNs with percentages similar to the ratios used in the F8BT:MEH-PPV:CN-PPV SPNs' synthesis were prepared and optically analyzed. Fig. 4C shows the absorption spectra of the mixture of the individual SPNs. The absorption spectra in the visible region were similar to those of the polyblend SPNs, however, they did not exhibit features associated with F8BT SPNs in the mixture except when their concentration in the mixture was dominating (i.e. $60 \%: 20 \%: 20 \%$ mixture).

Comparison of the emission spectra from the mixture of the individual SPNs (Fig. 4D) and the F8BT:MEH-PPV:CN-PPV SPNs (Fig. 4B) showed a marked difference, with F8BT's peak dominating in all nanoparticles' mixtures prepared. The emission peak broadened with the increase in the percentages of $\mathrm{MEH}$ PPV SPNs and CN-PPV SPNs, and had the widest broadening with the highest percentage of CN-PPV SPNs. Whilst referring to our previous work on these systems, ${ }^{5,16}$ we suggest that there were no interactions between the polymer nanoparticles when mixed, with each nanoparticulate species exhibiting their individual absorption and emissive characteristics.

The differences between the spectra of F8BT:MEH-PPV:CNPPV SPNs and the mixture of the individual SPNs in Fig. 4
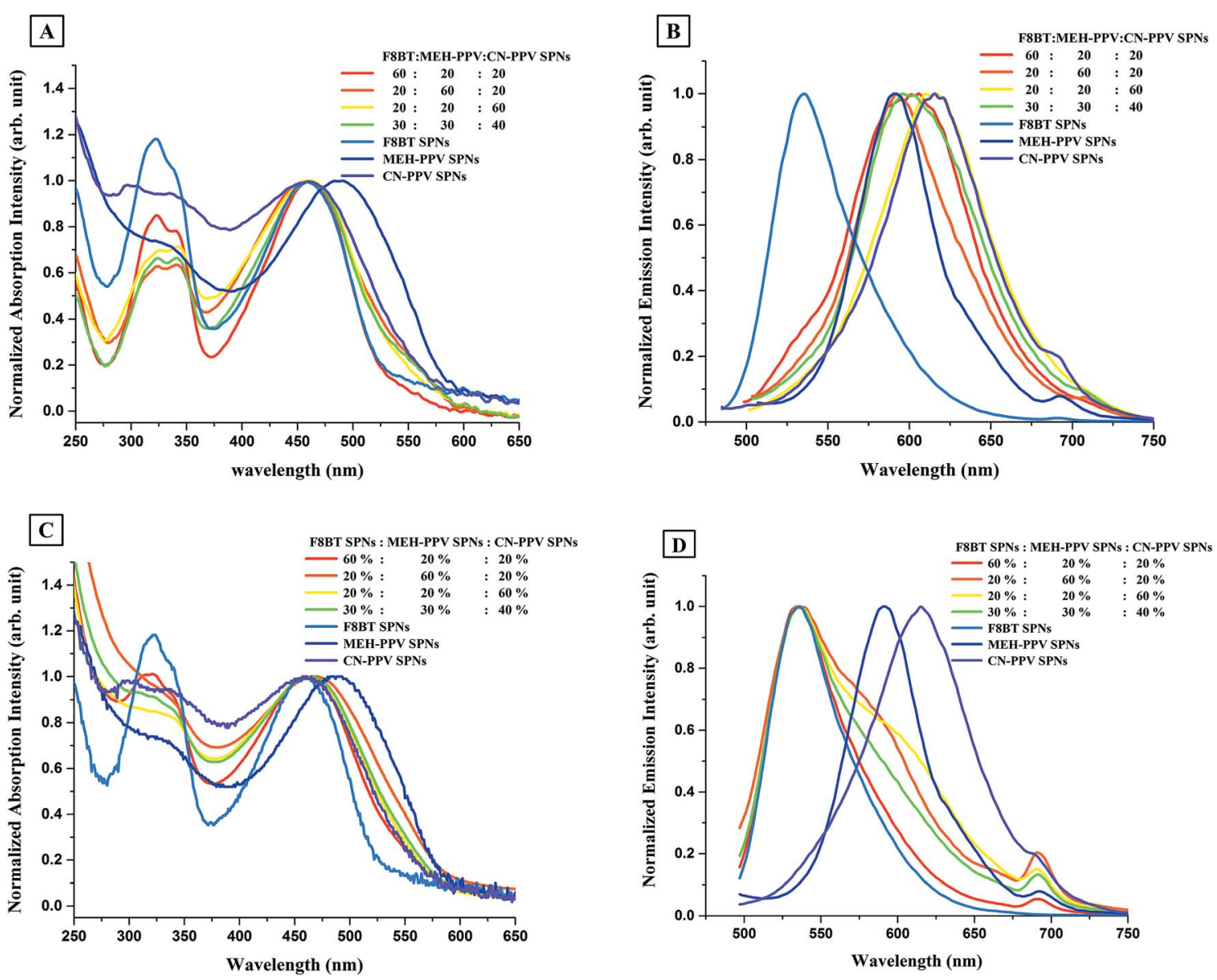

Fig. 4 The (A) absorption and (B) emission spectra of F8BT:MEH-PPV:CN-PPV SPNs, excitation wavelength $=460 \mathrm{~nm}$, and the (C) absorption and (D) emission of mixtures of the individual polymer SPNs, excitation wavelength $=460 \mathrm{~nm}$. The spectra of the individual polymer SPNs are also drawn for comparison. 
support our hypothesis of optically-observable electronic interactions between the polymers when brought together in one nanoparticulate configuration.

To understand this change in the optical properties of F8BT:MEH-PPV:CN-PPV SPNs and to identify which polymers interacted in this new configuration, one must first understand the inter-chain interactions between two polymers of the three when brought together in one-nanoparticle configuration. Fig. 5 shows the absorption and emission spectra of SPNs prepared from blends of F8BT and MEH-PPV with different ratios. The spectra of SPNs prepared from the individual polymers were also included for comparison. The absorption features of F8BT:MEH-PPV SPNs in the visible region (Fig. 5A), shifted slightly between the two constituent peaks of the SPNs made from their respective polymers, however the widths widened to cover both peaks. The shift was consistent and dependant on the ratios used, with the peaks shifting closer to the feature exhibited by the dominant polymer. There was also a change in the absorption spectra of F8BT:MEH-PPV SPNs in the UV region with the feature shape mostly resembling that of F8BT and decreasing in intensity with the decrease in its ratio. The emission spectra of F8BT:MEH-PPV SPNs with different polymer ratios (Fig. 5B) exhibited emission with spectra position, shape, and width consistent with pure MEH-PPV SPNs, with negligible variations. This was found to be the case even for SPNs which contained higher F8BT ratios despite the fact that F8BT SPNs were reported previously to have significantly higher quantum yields than MEH-PPV SPNs. ${ }^{\mathbf{1 6}}$ This optical behavior was explained previously ${ }^{\mathbf{9} 11}$ as an energy transfer from F8BT to MEH-PPV which can also be used to explain the disappearance of F8BT's emission peak, or the appearance of only a small F8BT related shoulder in F8BT:MEH-PPV:CN-PPV SPNs.

MEH-PPV was also blended with CN-PPV in the synthesis to produce MEH-PPV:CN-PPV SPNs. The absorption and emission spectra of the SPNs prepared from the polymers individually and from polyblends of the two polymers with different ratios are shown in Fig. 6. The absorption features of MEH-PPV:CNPPV SPNs in the visible region were similar to those of the
SPNs prepared from their constituent polymers, with the maxima of the visible-region's peak of seven of the nine SPNS prepared with different ratios coinciding with the maximum of CN-PPV SPNs, although this peak (ca. $460 \mathrm{~nm}$ ) was $\sim 32 \mathrm{~nm}$ blue-shifted from the peak of MEH-PPV SPNs (488 nm). The absorption maxima of the other two SPNs (70:30 and $30: 70$ MEH-PPV : CN-PPV SPNs) were slightly shifted to the right $\left(\lambda_{\mathrm{abs}, \max }=471 \mathrm{~nm}\right.$ for $\left.70: 30\right)$ or the left $\left(\lambda_{\mathrm{abs}, \max }=440 \mathrm{~nm}\right.$ for $30: 70)$ of the maximum of CN-PPV SPNs $\left(\lambda_{\mathrm{abs}, \max }=460 \mathrm{~nm}\right.$ for CN-PPV SPNs). Despite this overlapping of the peaks' absorption maxima in the visible region, their peak widths did not always match that of CN-PPV SPNs but decreased with the decrease in CN-PPV's ratio to match that of MEH-PPV SPNs. This consistent decrease in absorption width was also followed by a consistent decrease in the absorption intensity of the SPNS in the UV region $(320 \mathrm{~nm})$. The emission spectra of $\mathrm{MEH}-$ PPV:CN-PPV SPNs shifted to the blue region $(575 \mathrm{~nm})$ with the decrease in the ratio of CN-PPV. The blue-shift was observed a few nanometers before the peak of CN-PPV SPNs $\left(\lambda_{\text {em,max }}=\right.$ $617 \mathrm{~nm}$ for $10: 90 \mathrm{MEH}-\mathrm{PPV}: \mathrm{CN}-\mathrm{PPV}$ SPNs, $\lambda_{\mathrm{em}, \max }=615 \mathrm{~nm}$ for CN-PPV SPNs), and bypassed the peak of MEH-PPV SPNs by $17 \mathrm{~nm}\left(\lambda_{\text {em,max }}=575 \mathrm{~nm}\right.$ for $90: 10 \mathrm{MEH}-\mathrm{PPV}:$ CN-PPV SPNs, $\lambda_{\text {em,max }}=592 \mathrm{~nm}$ for MEH-PPV SPNs). This $42 \mathrm{~nm}$ blue-shift revealed a different interaction when compared to F8BT and MEH-PPV in the F8BT:MEH-PPV SPNs. A similar blue shift with the increase in MEH-PPV's ratio to CN-PPV was also visible in the emission spectra of F8BT:MEH-PPV:CN-PPV SPNs (Fig. 4B). This suggest that the shift in F8BT:MEH-PPV:CN-PPV SPNs' emission peak with the change in the polymers' ratios was caused by an inter-chain interaction between the MEH-PPV and CN-PPV polymers in the nanoparticles.

Fig. 6B also shows second small peaks in the red region of the spectra of MEH-PPV:CN-PPV SPNs, around $\lambda=691 \mathrm{~nm}$. These second peaks were also visible in the emission spectra of both MEH-PPV SPNs and CN-PPV SPNs and were found to be excitation wavelength dependant, as shown in Fig. 7A. Changing the concentration of the same SPNs and remeasuring their emission spectrum (under the same
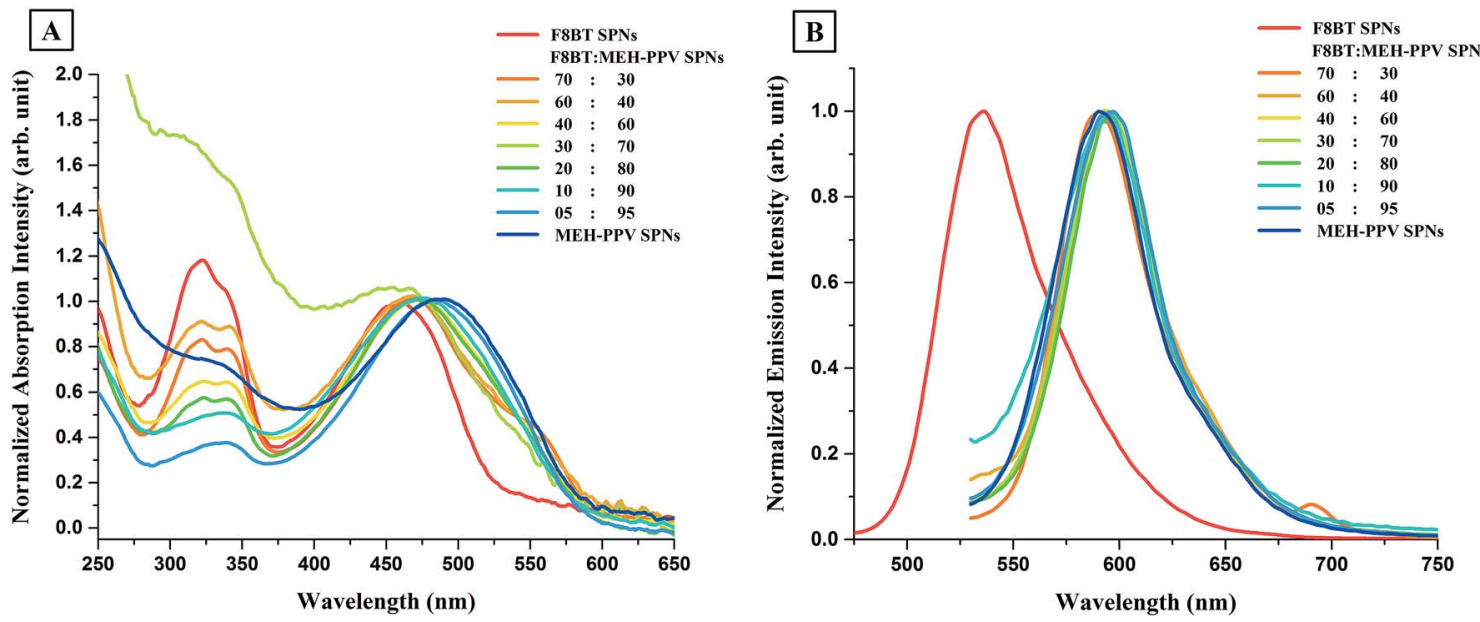

Fig. 5 The (A) absorption and (B) emission spectra of F8BT:MEH-PPV SPNs, excitation wavelength $=500 \mathrm{~nm}$ for all the samples containing MEHPPV and $460 \mathrm{~nm}$ for F8BT SPNs. The spectra of the individual polymer SPNs are also drawn for comparison. 

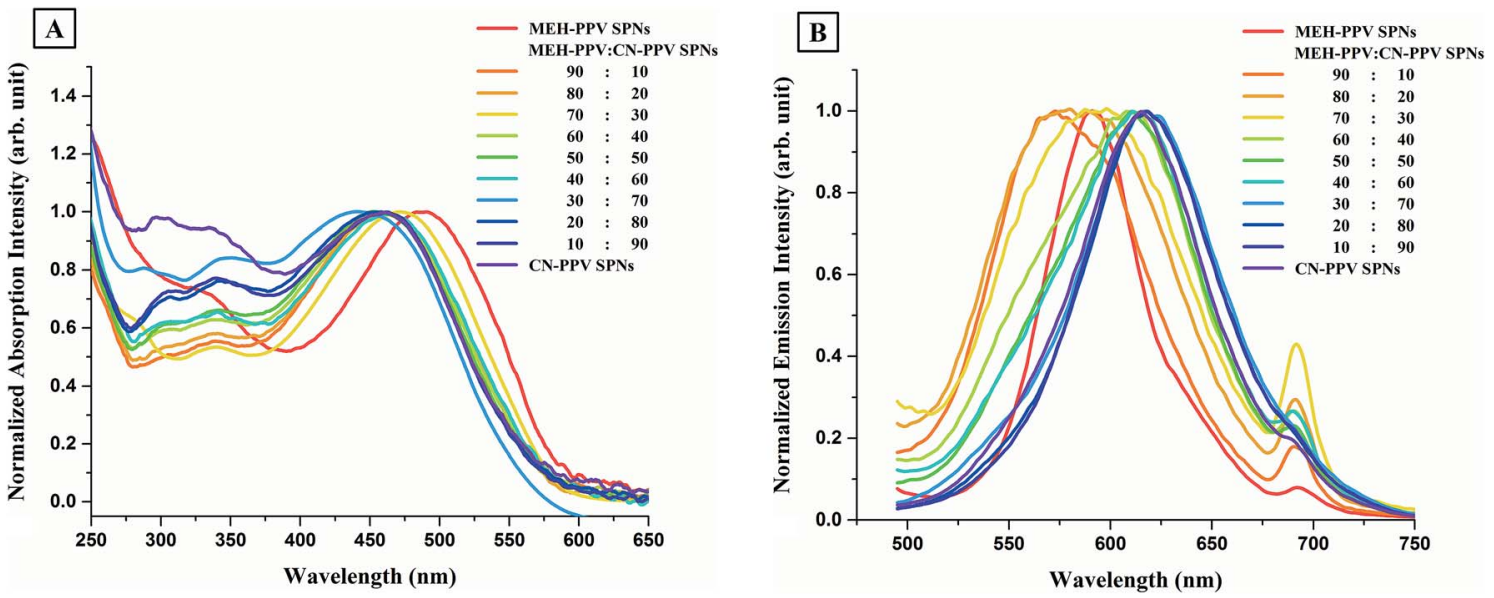

Fig. 6 The (A) absorption and (B) emission spectra of MEH-PPV:CN-PPV SPNs, excitation wavelength $=460 \mathrm{~nm}$. The spectra of the individual polymer SPNs are also drawn for comparison.
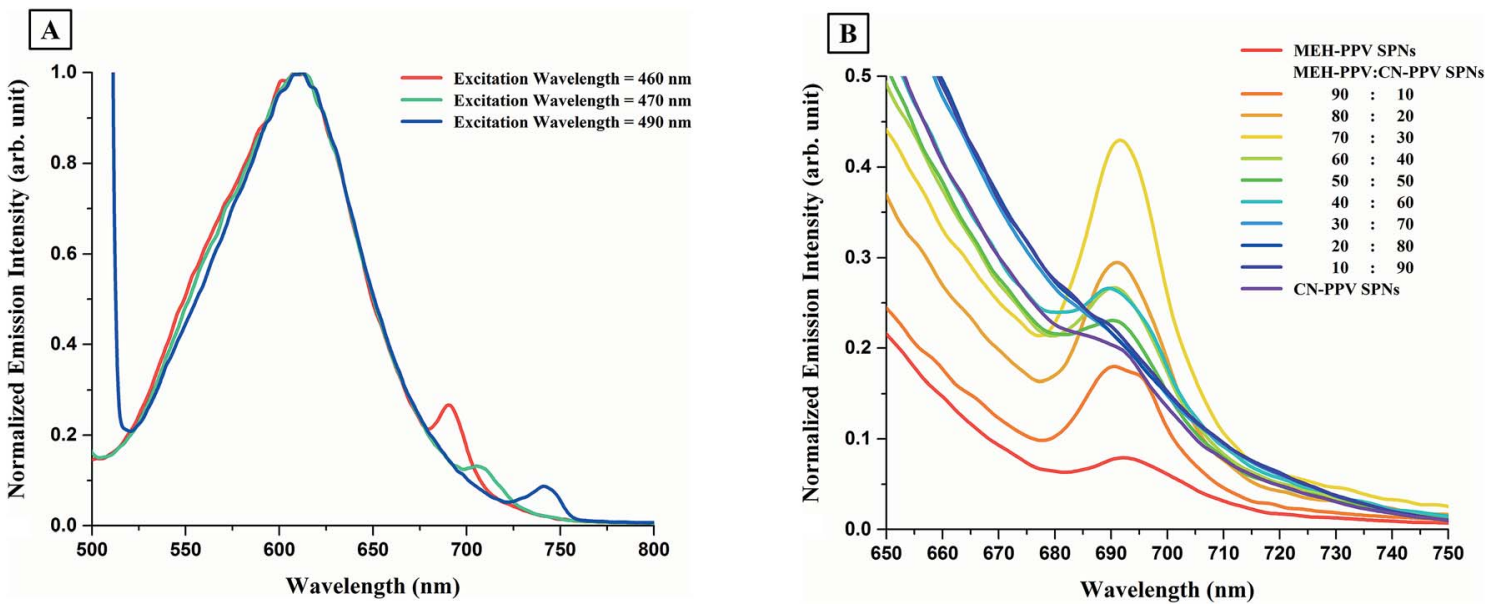

Fig. 7 The (A) emission spectra of $60: 40 \mathrm{MEH}-\mathrm{PPV}$ : CN-PPV SPNs under different excitation wavelengths, and (B) the emission spectra of MEH-PPV:CN-PPV SPNs zooming at the second peak, excitation wavelength $=460 \mathrm{~nm}$.

excitation wavelength) did not cause a change in the intensity or position of that second peak. However, constantly increasing the ratio of one of the polymers to the other in MEH-PPV:CNPPV SPNs caused a consistent change in its intensity, as shown in Fig. 7B.

CN-PPV was then blended with F8BT in different ratios, to produce F8BT:CN-PPV SPNs. Both polymers absorb in a similar visible region and their peak maxima lie at the same wavelength. The only difference between the absorption peaks of both polymers in the visible region is that the peak is broader for CN-PPV. Both polymers also absorb in the UV region however, F8BT has a more defined peak with a higher intensity than CN-PPV in that region. Fig. 8A shows the absorption spectra of the different F8BT:CN-PPV SPNs alongside the spectra of F8BT SPNs and CN-PPV SPNs. Decreasing the ratio of F8BT in the synthesis (from $90 \%$ to $10 \%$ ) resulted in the broadening of the SPNs' peaks in the visible region and the decrease in their UV absorption (which followed the shape of F8BT for ratios $90: 10$ to $30: 70$ and the shape of CN-PPV for ratios $20: 80$ and $10: 90$ ). This suggests that SPNs with low UV absorption can be synthesized from a blend of polymers which have high UV absorption. The variation in the bandwidth of the absorption peaks in the visible region and the decrease in the UV absorption of F8BT:CN-PPV SPNs with the decrease in F8BT's ratio in the SPNs was also observed in the absorption spectra of F8BT:MEH-PPV:CN-PPV SPNs (Fig. 4A).

The emission spectra of F8BTr:CN-PPV SPNs are shown in Fig. 8B. The blending of as little as $10 \%$ F8BT with CN-PPV resulted in the appearance of a shoulder on the left of the F8BT:CN-PPV SPNs' spectrum. The emission's peak of the SPNs synthesized from that specific blend was positioned exactly on the peak of CN-PPV SPNs, and its shoulder was positioned exactly where F8BT's emission peak was. Increasing F8BT's ratio and decreasing CN-PPV's ratio resulted in a consequent increase in F8BT's emission intensity and a consequent decrease in CN-PPV's emission intensity, and with F8BT ratios more that $40 \%$, F8BT's emission intensity became dominant and its peak became the main feature. This suggested that there 

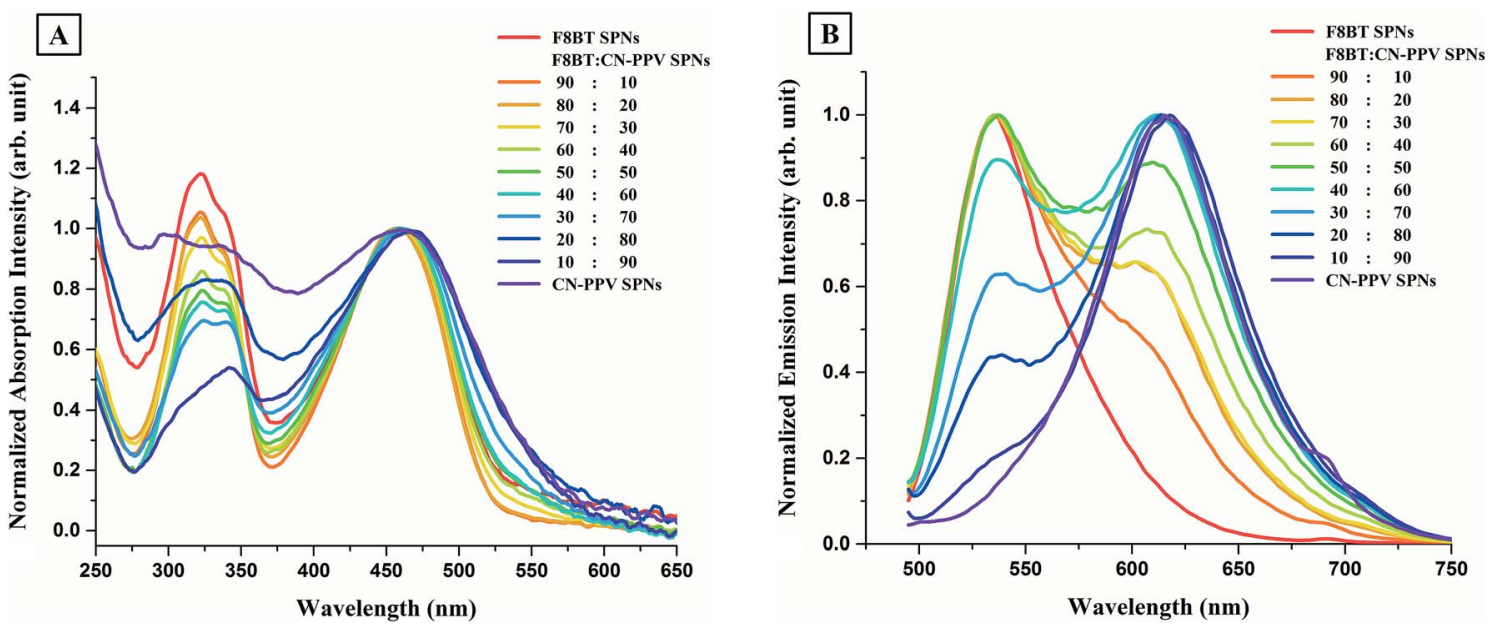

Fig. 8 The (A) absorption and (B) emission spectra of F8BT:CN-PPV SPNs, excitation wavelength $=460 \mathrm{~nm}$. The spectra of the individual polymer SPNs are also drawn for comparison.

were no inter-chain interactions between both polymers and the increase in the ratio of one resulted in a consequent increase in its emission intensity in the polyblend SPNs.

\section{Experimental}

The particles were prepared using the miniemulsion method reported previously, ${ }^{5,16}$ with some minor modifications to produce aqueous SPNs from a blend of three conjugated polymers with different ratios; two red/orange emitting polymers poly[2-methoxy-5-(2-ethylhexyloxy)-1,4-phenylenevinylene] (MEH-PPV, $M_{\mathrm{W}} 70$ 000-100 000, Sigma Aldrich) and poly(2,5di(hexyloxy)cyanoterephthalylidene) (CN-PPV, $M_{\mathrm{W}}$ not stated, Sigma Aldrich), and one green emitting polymer poly(9,9-dioctylfluorene-alt-benzothiadiazole) (F8BT, $M_{\mathrm{W}}$ 10,000-20 000, Sigma Aldrich). The SPNs prepared from the blend of the three polymers were called F8BT:MEH-PPV:CN-PPV SPNs and were prepared with weight ratios $(20: 20: 60),(20: 60: 20)$, $(60: 20: 20)$ and $(30: 30: 40)$. SPNs from the individual polymers and from the blends of two of the three polymers were also synthesized for comparison; F8BT SPNs, MEH-PPV SPNs, and CN-PPV SPNs, F8BT:MEH-PPV SPNs, MEH-PPV:CN-PPV SPNs, and F8BT:CN-PPV SPNs. The SPNs synthesized from the blend of two polymers were prepared with nine different polymer weight ratios; (10:90), (20:80), (30:70), (40:60), (50:50), (60:40), (70:30), $(80: 20)$, and $(90: 10)$. The four different F8BT:MEH-PPV:CN-PPV SPNs were also compared with four mixtures of the pre-synthesized individual SPNs prepared with percentages equal to their ratios.

In this synthesis, the solvent dichloromethane was substituted with chloroform due to its lower boiling point. The surfactant SDS was used due to its higher emulsion stability when compared to poly-ethylene glycol (PEG), its abundance, ease of transport, and significantly cheaper price compared to phospholipids or other PEG based surfactants despite the fact that SDS is not intended for medical applications. ${ }^{17}$
In a typical synthesis, $15 \mathrm{mg}$ of each polymer was dissolved in $15 \mathrm{~mL}$ chloroform to form polymer stock solutions. In another flask, $43 \mathrm{mg}$ sodium dodecyl sulfate (SDS) was dissolved in $25 \mathrm{~mL}$ of ultra-pure water. Then, $1 \mathrm{~mL}$ of the polymer stock solution was introduced over a period of 60 seconds, and the emulsion was stirred for 10 minutes, sonicated for 2 minutes at 7 degrees celsius, then stirred gently again for 5 minutes. The milky suspension was then stirred vigorously overnight to ensure the evaporation of the chloroform and formation of the nanoparticles. For the polyblend SPNs; the introduced $1 \mathrm{~mL}$ of polymer solution was first prepared by mixing ratios of the polymers, for example, for $20: 20: 60$ F8BT : MEH-PPV : CN-PPV SPNs; $400 \mu \mathrm{L}$ F8BT stock solution, $400 \mu \mathrm{L}$ MEH-PPV stock solution, and $1200 \mu \mathrm{L}$ CN-PPV stock solution were mixed and $1 \mathrm{~mL}$ of the resulting solution was used in the synthesis.

The resultant nanoparticles' shapes and sizes were determined by transmission electron microscopy (TEM, JEM-1011, JEOL), and their optical properties were determined by absorption spectrometry (Thermo Scientific, Genesys 10S UVVis Spectrometer) and emission spectrometry (Perkin Elmer Luminescence Spectrometer, LS45).

\section{Conclusions}

In conclusion, F8BT:MEH-PPV:CN-PPV SPNs were synthesized and their optical spectra were found to be different than those of mixtures of the individual polymer SPNs due to inter-chain interactions between MEH-PPV and F8BT and between MEHPPV and CN-PPV. Notably, there were no direct optically observed inter-chain interactions between the chains of F8BT and CN-PPV in terms of emission, however, mixing both polymers in one nanoparticle caused a modification in the particles' UV absorption. With the inclusion of the three polymers within one nanoparticle, multiple inter-chain interactions occurred between MEH-PPV and the other polymers facilitating an indirect interaction between the chains of F8BT and CN-PPV. 


\section{Conflicts of interest}

There are no conflicts to declare.

\section{Acknowledgements}

We acknowledge Dr Salim M. El-Hamidy, Electronic Microscopy Unit, Biological Science Department, Faculty of Sciences, KAU, for TEM assistance.

\section{Notes and references}

1 Z. Hashim, PhD thesis, King's College London, 2013.

2 P. Howes, M. Green, A. Bowers, D. Parker, G. Varma, M. Kallumadil, M. Hughes, A. Warley, A. Brain and R. Botnar, J. Am. Chem. Soc., 2010, 132, 9833.

3 J. Pichaandi and F. C. J. M. van Veggel, Coord. Chem. Rev., 2014, 263-264, 138.

4 C. Philippot and P. Reiss, in Frontiers of Nanoscience, ed. M. d. l. F. Jesus and V. Grazu, Elsevier, 2012, vol. 4, p. 81.

5 Z. Hashim, P. Howes and M. Green, J. Mater. Chem., 2011, 21, 1797.

6 P. Howes and M. Green, Photochem. Photobiol. Sci., 2010, 9, 1159.
7 E. Kemal, T. F. Abelha, L. Urbano, R. Peters, D. M. Owen, P. Howes, M. Green and L. A. Dailey, RSC Adv., 2017, 7, 15255.

8 Y. Q. Ge, Y. Zhang, S. Y. He, F. Nie, G. J. Teng and N. Gu, Nanoscale Res. Lett., 2009, 4, 287-295.

9 C. F. Wu, H. S. Peng, Y. F. Jiang and J. McNeill, J. Phys. Chem. $B, 2006,110,14148$.

10 D. Feldman, J. Macromol. Sci., Part A: Pure Appl.Chem., 2015, 52, 648.

11 X. Wang, L. C. Groff and J. D. McNeill, J. Phys. Chem. C, 2014, 118, 25731.

12 C. F. Huebner, R. D. Roeder and S. H. Foulger, Adv. Funct. Mater., 2009, 19, 3604.

13 H. Yabu, Polym. J., 2013, 45, 261.

14 M.-S. Jang, S.-Y. Song, H.-K. Shim, T. Zyung, S.-D. Jung and L.-M. Do, Synth. Met., 1997, 91, 317.

15 T. Higuchi, A. Tajima, H. Yabu and M. Shimomura, Soft Matter, 2008, 4, 1302.

16 Z. Hashim, M. Green, P. H. Chung, K. Suhling, A. Protti, A. Phinikaridou, R. Botnar, R. A. Khanbeigi, M. Thanou, L. A. Dailey, N. J. Commander, C. Rowland, J. Scott and D. Jenner, Nanoscale, 2014, 6, 8376.

17 M. Green, P. Howes, C. Berry, O. Argyros and M. Thanou, Proc. R. Soc. A, 2009, 465, 2751. 